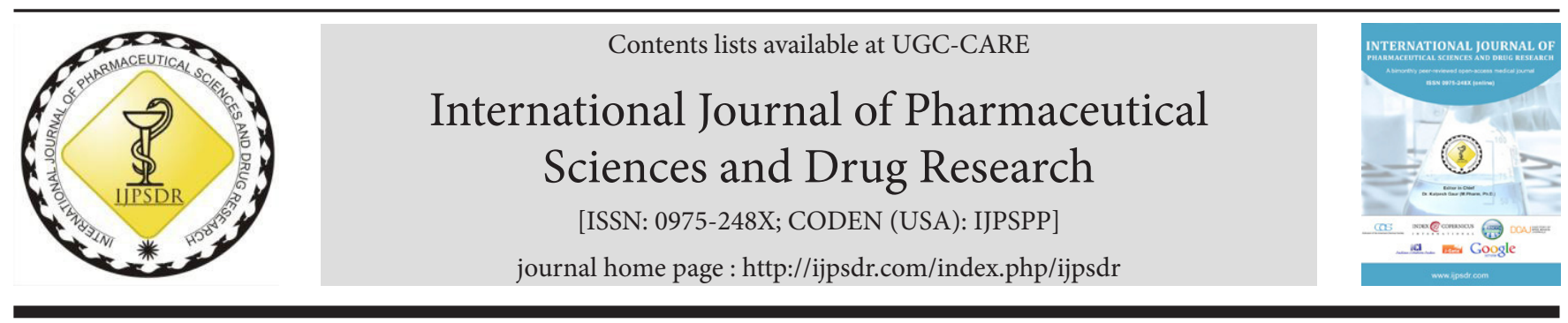

Research Article

\title{
In situ Nasal Gel of Granisetron for Enhancement of Bioavailability over Oral Delivery: Formulation, Optimization, and In vivo Evaluation
}

\author{
Rahul Padalkar*, Ashwini Madgulkar \\ AISSMS College of Pharmacy, Kennedy Road, Near RTO, Pune-411001, Maharashtra, India
}

\author{
ART I CLE INFO \\ Article history: \\ Received: 29 June, 2020 \\ Revised: 13 August, 2020 \\ Accepted: 22 August, 2020 \\ Published: 30 September, 2020 \\ Keywords: \\ Box-Behnken design, Fulvic acid, \\ Granisetron, In situ nasal gel, \\ Poloxamer PF 127, \\ Transmucosal. \\ DOI: \\ 10.25004/IJPSDR.2020.120517
}

\begin{abstract}
A B S T R A C T
The objective of the present work was the formulation, optimization, and in vivo evaluation of in situ nasal gel of granisetron (GRA) that shows liquid to gel transformation at nasal temperature $\left(32-34^{\circ} \mathrm{C}\right)$ and maximum drug release after 4 hours; shows bioavailability enhancement over oral delivery. Formulations were prepared using poloxamer PF 127 as gel-forming polymer, carbopol as a mucoadhesive agent, and fulvic acid as a penetration enhancer. A Box-Behnken design was used to prepare the experimental batches and Design Expert software for optimization of the formulation. Ex vivo evaluations were carried out on sheep nasal mucosa and for in vivo evaluation, rabbits were used. It was observed that optimized formulation showed gelation temperature near $33^{\circ} \mathrm{C}$ and drug release of $96 \%$ after 4 hours. Fulvic acid was evaluated as a penetration enhancer in this work and showed significant enhancement of drug diffusion across the nasal mucosal membrane. Ex vivo histological evaluation of nasal mucosa treated with optimized formulation showed no significant destructive effects. In vivo evaluations showed that the plasma level profile of prepared in situ nasal gel was enhanced significantly over oral delivery. The findings suggested that nasal route nasal transmucosal delivery of GRA can result in enhancement of its bioavailability over the oral route.
\end{abstract}

\section{INTRODUCTION}

The GRA is a potent 5-HT3 receptor antagonist, widely prescribed for the prevention and treatment of chemotherapy-induced nausea and vomiting. Although GRA is rapidly absorbed from the gastrointestinal tract, it is subjected to extensive first-pass metabolism. The oral bioavailability of GRA is about 41 to $60 \%$. In healthy volunteers, a half-life of about 3 to 4 hours is reported. ${ }^{[1,2]}$ Therefore, current GRA treatment generally involves an oral dose of 1 to $2 \mathrm{mg}$ within 1-hour before the start of chemotherapy treatment, then $2 \mathrm{mg}$ daily in 1 to 2 divided doses up to 4 days. Also, a single $3 \mathrm{mg}$ IV dose of GRA can be administered, repeated if necessary with a maximum daily dose of $9 \mathrm{mg}^{[1,3]}$ Although GRA is effective when given orally, it has got many limitations. Once vomiting has started, particularly if it is moderate or severe, the oral route clearly cannot be used and some alternative is needed. The most common parenteral alternatives to the oral route are injectables. The limitations of these dosage forms include pain and the need for a skilled person for administration because of their invasive nature. In order to improve patient acceptance, there is a need to design a dosage form that offers the advantages of injectables, like the rapid onset of action, improved bioavailability, and simultaneously it should allow self-administration and be non-invasive.

The transmucosal route is being explored as a noninvasive alternative to injectable parenteral routes. ${ }^{[4,5]}$ The mucous membrane is present at various sites, like the nasal cavity, buccal cavity, git, rectum, vagina, eyes, etc.

\footnotetext{
${ }^{*}$ Corresponding Author: Rahul Padalkar

Address: AISSMS College of Pharmacy, Kennedy Road, Near RTO, Pune-411001, Maharashtra, India

Email $\bowtie$ : rahulvita@gmail.com

Relevant conflicts of interest/financial disclosures: The authors declare that the research was conducted in the absence of any commercial or financial relationships that could be construed as a potential conflict of interest.

Copyright (C) 2020 Rahul Padalkar et al. This is an open access article distributed under the terms of the Creative Commons Attribution- NonCommercialShareAlike 4.0 International License which allows others to remix, tweak, and build upon the work non-commercially, as long as the author is credited and the new creations are licensed under the identical terms.
} 
Amongst these, nasal mucosa has distinct advantages. The nasal route is a non-invasive, painless procedure, does not require hospitalization, and hence, could be accessible to a wide range of patient populations and a highly acceptable option for drug dosing. A high degree of vascularization and permeability of the nasal mucosa makes it an attractive site for drug delivery. The use of an effective multiday nasal therapeutic system of GRA can be expected to facilitate antiemetic therapy success.

Various dosage forms have been explored for nasal delivery. Dry powder inhalers can induce sneezing or irritation to the mucosa. This is less observed with liquids administered as drops or sprays. But they may be drained off towards oropharynx or nasal openings due to lack of enough viscosity and mucoadhesion. A viscous dosage form would again be difficult to handle and administer. Therefore, there is a need for a dosage form that is liquid during storage and administration but becomes viscous and mucoadhesive on the nasal mucosa. In situ nasal gels offer this advantage. When stored under appropriate conditions, they are non-viscous and suddenly get transformed into a gel upon contact with nasal mucosa. ${ }^{[6]}$ Thus, they avoid washout due to various factors and prolonged residence time on the mucosa, thereby favoring the absorption of the drug.

Penetration enhancers play a crucial role in improving drug flux across biological membranes, including gastric epithelial or nasal membrane. ${ }^{[7]}$ The absorption of hydrophilic drugs across the mucus membrane can be enhanced by opening the tight junctions between cells and increasing the vascularity at the basal membrane of the cells. In the present work, fulvic acid has been investigated as a possible penetration enhancer for GRA across the nasal mucosa. Fulvic acid is a highly water-soluble, naturally occurring substance commonly isolated from soil and plants. Very few works using fulvic acid as an excipient have been reported to date. ${ }^{[8,9]}$ Fulvic acid has been explored in the presented work as a transmucosal permeation enhancer. The proposed work aims to achieve plasma levels of GRA through nasal in situ gel, which shows enhancement over oral drug delivery.

\section{MATERIALS AND METHODS}

\section{Materials}

The GRA was gifted by Wockhardt Research Centre (Aurangabad, India). Graniset (GRA HCl i.v. $1 \mathrm{mg} / \mathrm{mL}$ marketed formulation) was purchased from a local pharmacy. Poloxamer (PF 127) and benzalkonium chloride were purchased from Signet Chemical Corporation (Mumbai, India). Fulvic acid was supplied as a gift sample by Greenchem Biotech (Pune, India). Carbopol 934 was purchased from Loba Chemicals (Mumbai, India) and all other reagents were commercially analytical-grade.

\section{Preparation of In situ Nasal Gels}

The in situ nasal gels were prepared using the cold method. ${ }^{[10]}$ PF 127 was selected as a gel-forming polymer. Carbopol was selected as a mucoadhesive polymer. Fulvic acid was used as a penetration enhancer. The method involved the slow addition of PF 127 in cold water with continuous agitation. Then drug, fulvic acid, carbopol 974P, and benzalkonium chloride (as a preservative) were added with continuous agitation. The formed mixtures were stored overnight at $4^{\circ} \mathrm{C}$ until a clear and transparent solution was formed. Finally, the volume was adjusted by using cold distilled water.

\section{Experimental Design}

From the pre-formulation studies, it was observed that concentration of poloxamer, carbopol, and fulvic acid was influencing gelation temperature and drug diffusion across sheep nasal mucosa. It was necessary to investigate the influence of the individual formulation factor on these response variables. Thus, a statistical design of the experiment was necessary. When the experimental design has three formulation factors, the Box-Behnken design requires fewer runs (i.e.,12) and reduces the number of experiments as compared to a $3^{3}$ factorial design that involves 27 runs. Therefore, batches for in situ nasal gel were prepared using a $3^{3}$ Box-Behnken design. ${ }^{[11,12]}$ The composition of test formulations as per the experimental design is shown in Table 1.

\section{Evaluation of In situ Nasal Gels}

\section{Content of GRA}

A $1 \mathrm{~mL}$ of the formulation was taken in a $100 \mathrm{~mL}$ volumetric flask, and then the volume was made to $100 \mathrm{~mL}$ with distilled water. ${ }^{[13]}$ This solution was further diluted 50 times and the resultant solution was analyzed by ultraviolet (UV)-visible spectrophotometry $\left(\lambda_{\max }=302 \mathrm{~nm}\right.$ for GRA).

\section{Determination of Gelation Temperature}

Gelation and gel melting was assessed using a modification of the Miller and Donavan technique. ${ }^{[14]} \mathrm{A} 2 \mathrm{~mL}$ aliquot of the gel was transferred to test tubes, immersed in a water bath at $4^{\circ} \mathrm{C}$. The temperature of the water circulation bath, as shown in Fig. 1, was increased in increments of $1^{\circ} \mathrm{C}$ per minute, and left to equilibrate for 5 minutes at each new setting. The samples were then examined for gelation, which was said to have occurred when the meniscus would no longer move upon tilting through $180^{\circ}$, as shown in Fig. 1. The upper test tube on the right side of Fig. 3 shows that liquid fell on inverting the test-tube,
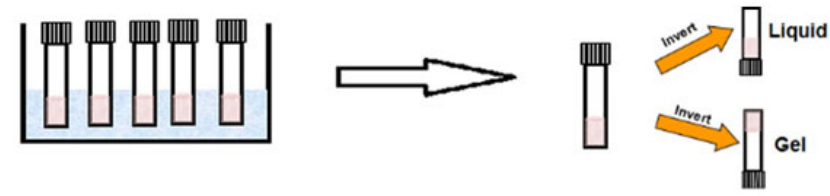

Fig. 1: Measurement of gelation temperature 
Rahul Padalkar et al.

Table 1: Composition of in situ nasal gel as per Box-Benhken design

\begin{tabular}{|c|c|c|c|c|c|}
\hline Formulation code & $\begin{array}{l}\text { Poloxamer } 403 \\
\% w / v\end{array}$ & Carbopol \%w/v & $\begin{array}{l}\text { Fulvic acid } \\
\% w / v\end{array}$ & $\begin{array}{l}\text { Benzalkonium chloride } \\
\% \mathrm{v} / \mathrm{v}\end{array}$ & Purified water $(\mathrm{mL})$ \\
\hline $\mathrm{F}_{1}$ & 14 & 0.1 & 0.5 & 0.5 & q.s. 10 \\
\hline $\mathrm{F}_{2}$ & 14 & 0.5 & 0.5 & 0.5 & q.s. 10 \\
\hline $\mathrm{F}_{3}$ & 20 & 0.1 & 0.5 & 0.5 & q.s. 10 \\
\hline $\mathrm{F}_{4}$ & 20 & 0.5 & 0.5 & 0.5 & q.s. 10 \\
\hline $\mathrm{F}_{5}$ & 17 & 0.1 & 0.25 & 0.5 & q.s. 10 \\
\hline $\mathrm{F}_{6}$ & 17 & 0.1 & 1 & 0.5 & q.s. 10 \\
\hline $\mathrm{F}_{7}$ & 17 & 0.5 & 0.25 & 0.5 & q.s. 10 \\
\hline $\mathrm{F}_{8}$ & 17 & 0.5 & 1 & 0.5 & q.s. 10 \\
\hline $\mathrm{F}_{9}$ & 14 & 0.3 & 0.25 & 0.5 & q.s. 10 \\
\hline $\mathrm{F}_{10}$ & 20 & 0.3 & 0.25 & 0.5 & q.s. 10 \\
\hline $\mathrm{F}_{11}$ & 14 & 0.3 & 1 & 0.5 & q.s. 10 \\
\hline $\mathrm{F}_{12}$ & 20 & 0.3 & 1 & 0.5 & q.s. 10 \\
\hline
\end{tabular}

due to lack of gelation, whereas the lower test tube shows that the liquid did not fall down on inverting the tube due to gel formation.

\section{Viscosity Measurement}

The viscosity of formulations was measured using the cone and plate viscometer model. The sample at low temperature was placed on a viscometer. The temperature of the sample was raised above $40^{\circ} \mathrm{C}$, using a circulation bath. The sample was allowed to cool and the viscosity at various temperatures was recorded.

\section{Spreadability}

To measure the spreadability, the sheep nasal mucosa was taken in a beaker containing water that was maintained at the temperature of $35^{\circ} \mathrm{C}$ for 15 minutes by slow heating. The mucosa was taken on a glass slide and $0.5 \mathrm{~mL}$ of the formulation was added to it. The slide was tilted through $90^{\circ}$ and the distance traveled by the formulation was measured. Measurements were repeated three times for each of the gel preparation. ${ }^{[15]}$

\section{Ex vivo Study}

Fresh nasal tissues were carefully removed from the nasal cavity of sheep obtained from the local slaughterhouse. Tissue samples were mounted on Franz diffusion cell, in which $7 \mathrm{~mL}$ of phosphate buffer of $\mathrm{pH} 6.6$ at $34^{\circ} \mathrm{C}$ was added to the acceptor chamber. The temperature was maintained at $34^{\circ} \mathrm{C}$. After a pre-incubation time of 20 minutes, a formulation equivalent to $10 \mathrm{mg}$ of GRA was placed in the donor chamber. At predetermined time points, aliquot equal to $0.5 \mathrm{~mL}$ samples were withdrawn from the acceptor compartment, replacing the sampled volume with phosphate buffer of $\mathrm{pH} 6.6$ after each sampling, for a period of 240 minutes. The samples withdrawn were filtered and used for analysis. The amount of permeated drug was determined spectrophotometrically at $302 \mathrm{~nm}$. Permeability coefficient (P) was calculated from the slope

of the graph of the percentage of drug transported $v s$. time and shown in equation I. Flux and enhancement ratio was calculated from equations II and III.

$$
\mathrm{P}=\text { slope } \times \mathrm{Vd} / \mathrm{S}
$$

Where, $\mathrm{Vd}=$ Volume of donor solution, $\mathrm{S}=$ Surface area of tissue

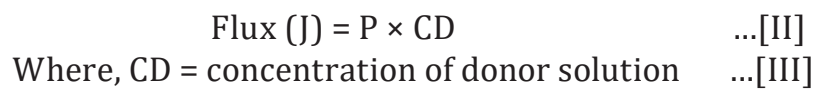

\section{Optimization}

The objective was to obtain optimum concentrations of poloxamer, carbopol, and fulvic acid to formulate an in situ nasal gel of GRA that shows gelation at a temperature as close as possible to 32 to $34^{\circ} \mathrm{C}$ and maximum cumulative drug release after 4 hours. This criterion was fed to the software after analysis of variance (ANOVA) and the optimum formulation was searched.

\section{Histological Evaluation of Nasal Mucosa}

Histological study of the excised nasal mucosa was conducted by comparing a fresh mucosa treated with phosphate buffer, drug solution, and optimized formulation for 240 minutes in the ex vivo permeation study. ${ }^{[16]}$ The objective was to detect if any significant histological changes have occurred during the experiment. After permeation study, nasal mucosa was cleared off the gel, sectioned with a rotary microtome (Model 1090 A, The Western Electric and Scientific Works, India), and fixed in $10 \%$ formalin solution.

The sectioned tissue was then stained with hematoxylin and eosin. Mucosa treated with drug solution and phosphate buffer were treated similarly. Tissue sections were observed under an Olympus CKX41 optical microscope (Olympus Optical Co. Ltd., Tokyo, Japan).

\section{In vivo Bioavailability Studies}

The objective of these studies was to compare the bioavailability of GRA oral solution and its optimized in situ 
nasal gel. The optimized GRA in situ nasal gel formulation was selected for in vivo studies. Twelve white rabbits with an average weight of three kilograms were divided into three groups. All the experimental procedures and protocols used in this study were reviewed and approved by the Institutional Animal Ethical Committee (IAEC) of AISSMS College of Pharmacy, Pune, constituted under the Committee for Purpose of Control and Supervision of Experiments on Animals (CPCSEA), Ministry of Environment and Forests, Government of India. In the study, with 1 week apart as a wash-out period, $1 \mathrm{~mL}$ of an aqueous solution containing $4 \mathrm{mg}$ of GRA was given orally to the first group. The second group received $0.16 \mathrm{~mL}$ gel equivalent to $2 \mathrm{mg} / \mathrm{kg}$ GRA into each nostril of the rabbit, using a micropipette inserted $1 \mathrm{~cm}$ into the nostril. After administration of the different formulations, blood samples $(0.5 \mathrm{~mL})$ were collected at time intervals of 0,15 , $30,60,90,120,150,180,210$, and 240 minutes from the marginal ear vein of the rabbits.

\section{Evaluation of Pharmacokinetic Parameters}

\section{Standard Calibration Curve of GRA in Plasma}

Chromatographic conditions: The separation was achieved on a Nova-Pak C8, $4 \mu \mathrm{m}$ column $(250 \times 4.6 \mathrm{~mm})$. The isocratic mobile phase pumped at room temperature, at a flow rate of $1 \mathrm{~mL} / \mathrm{min}$, was a mixture of acetonitrile and $25 \mathrm{~mm}$ potassium dihydrogen phosphate (75:25, $\mathrm{v} / \mathrm{v}){ }^{[17,18]}$ The mobile phase was degassed by filtration through a $0.45 \mu \mathrm{m}$ filter and ultrasonication for 20 minutes. Detection was performed at $302 \mathrm{~nm}$.

Determination of plasma levels: Pharmacokinetics of GRA in rabbits was studied using its oral solution and optimized in situ nasal gel. Blood samples $(0.5 \mathrm{~mL})$ were collected in sterile test tubes containing tri-potassium EDTA as an anticoagulant. Collected blood samples were vortexed at 3,000 rpm for 20 minutes. The upper clear layer of plasma was carefully removed and mixed with $1 \mathrm{~mL}$ of acetonitrile to precipitate proteins. The mixture was again vortexed, filtered, and injected into an HPLC system. Peak areas for each sample were calculated and the following pharmacokinetic parameters were estimated.

- Peak plasma concentration $\left(\mathrm{C}_{\text {max }}\right)$ : expressed as $\mathrm{ng} / \mathrm{mL}$

- Time of peak plasma concentration $\left(t_{\text {max }}\right)$ : expressed in minutes

- Area under the curve (AUC): expressed as ng.hr/mL AUC represents the total integrated area under the plasma level-time profile and expresses the total amount of drug that comes into the systemic circulation after its administration. AUC was calculated by the trapezoidal rule.

\section{RESULTS}

\section{Evaluation of In situ Nasal Gel Formulations}

\section{Drug Content and $p H$ of Formulation}

The percentage drug content of all prepared nasal formulations was found to be in the range of 98 to $101 \%$. The $\mathrm{pH}$ of all formulations was found to be in the range of 4.4 to 6.6 (Table 2).

\section{Gelation Temperature}

The gelation temperature of test formulations ranged from 20 to $43.7^{\circ} \mathrm{C}$. The nature of the effect of each formulation variable on gelation temperature is discussed later in this text with the help of response surface plots.

\section{Spreadability}

The spreadability was found to get decreased with an increase in the concentration of carbopol. Comparison of formulations F1 and F2 (except carbopol, other variables are at fixed level) show that an increase in the concentration of carbopol decreased the spreadability. Comparison of formulations F9 and F10 (except poloxamer, other variables are at fixed level) showed that poloxamer also decreased the spreadability with increasing concentration

Table 2: Evaluation of in situ gel formulations

\begin{tabular}{llllll}
\hline Formulation & Drug content (\%) & $p H$ & Gelation temperature $\left({ }^{\circ} \mathrm{C}\right)$ & Ex vivo \% drug diffusion & Spreadability $(\mathrm{mm})$ \\
\hline F1 & 99.82 & 6.6 & 43.7 & 95.62 & 11 \\
F2 & 99.61 & 6.12 & 35.9 & 92.32 & 3 \\
F3 & 100.06 & 6.49 & 31.2 & 91.61 & 9 \\
F4 & 98.41 & 6 & 20 & 75.92 & 1 \\
F5 & 99 & 5.09 & 38 & 76.3 & 10 \\
F6 & 99.56 & 5.72 & 38.4 & 95.4 & 10 \\
F7 & 98.24 & 5.45 & 24.1 & 70.3 & 2 \\
F8 & 98.36 & 5.62 & 24.4 & 84.2 & 2 \\
F9 & 99.49 & 5.7 & 41.1 & 75.7 & 6 \\
F10 & 99.38 & 5.3 & 28.2 & 72.1 & 5 \\
F11 & 101 & 4.4 & 40.6 & 94.01 & 6 \\
F12 & 99.61 & 5.6 & 28 & 89.8 & 5 \\
\hline
\end{tabular}


but the magnitude of the effect was less as compared to carbopol. An increase in viscosity of formulation with an increase in poloxamer concentration contributes to this effect. But the mucoadhesive properties of carbopol were dominant over poloxamer for restricting the spreading of the gel. From F5 and F6, we can observe that there no significant effect of fulvic acid on the spreadability.

\section{Viscosity}

The viscosities of all formulations in the temperature range of 21 to $40^{\circ} \mathrm{C}$ are shown in Fig. 2. It was observed that

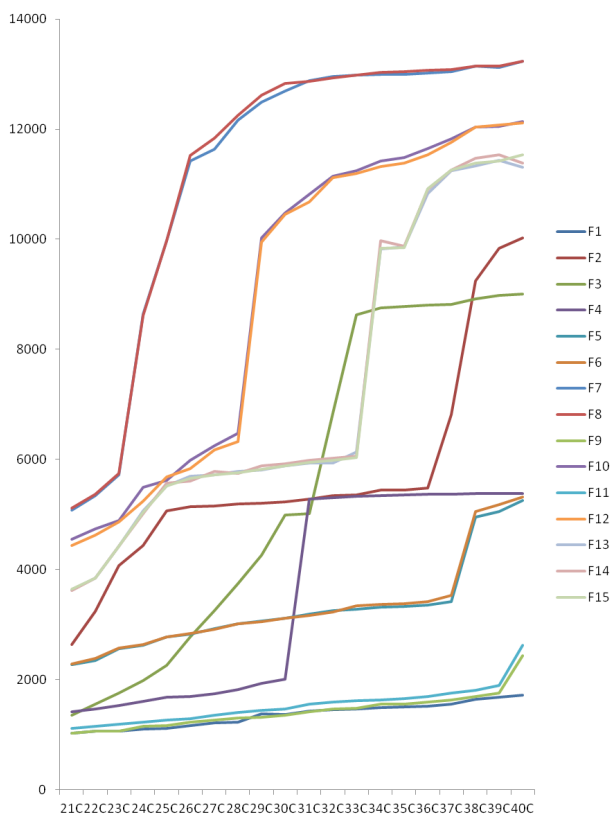

Fig. 2: Viscosity of formulations in temperature range of 21 to $40^{\circ} \mathrm{C}$

Table 3: Drug permeation profile of GRA in presence and absence of fulvic acid

\begin{tabular}{lll}
\hline & \multicolumn{2}{c}{ Batch } \\
\cline { 2 - 3 } Permeation profile & $\begin{array}{l}\text { In presence of fulvic } \\
\text { acid }\end{array}$ & $\begin{array}{l}\text { In absence of fulvic } \\
\text { acid }\end{array}$ \\
\hline $\begin{array}{l}\text { Permeability } \\
\text { coefficient } \\
(\mathrm{mg} . \mathrm{cm} / \mathrm{min})\end{array}$ & 0.1016 & 0.0376 \\
$\begin{array}{l}\text { Flux } \\
\left(\mathrm{mg} / \mathrm{cm}^{2} / \mathrm{min}\right)\end{array}$ & 10.03 & 0.474 \\
$\begin{array}{l}\text { Enhancement ratio } \\
(\mathrm{ER})\end{array}$ & 2.116 & - \\
\hline
\end{tabular}

viscosity increased with an increase in the concentration of poloxamer. Viscosity also increased with an increase in the concentration of carbopol. While there was no effect of fulvic acid on the viscosity of the formulation, there was a considerable change in viscosity at the point of gelation temperature as shown in Fig. 2. The gelation temperature can also be considered as the temperature at which a sudden rise in viscosity is observed. There was no significant effect of fulvic acid on the viscosity of formulations.

\section{Ex vivo Diffusion Study}

Ex vivo diffusion studies were carried out on sheep nasal mucosa on all formulations, which are shown in Table 2. The effect of poloxamer concentration, carbopol concentration, and fulvic acid concentration was studied on sheep nasal mucosa. From formulation F1 and F2, it was observed that as the concentration of carbopol increased the drug release decreased. The negative effect was due to an increase in the viscosity of the formulations. The same effect was observed in the case of poloxamer (F9 and F10), as the concentration of poloxamer increases the drug diffusion decreases, ${ }^{[19-21]}$ while formulation F5 and F6 showed a large positive effect of fulvic acid on drug release. Table 3 summarizes the effect of formulation components on diffusion flux.

\section{Statistical Models}

Analysis of experimental results was carried out by using Design-Expert software. After filling the data in the design, a quadratic model was suggested to run the design. F, $\mathrm{p}$ values, and model $\mathrm{F}$ value for gelation temperature and in vitro drug release were obtained from ANOVA (Table 4).

For gelation temperature response, the model $\mathrm{F}$ value of 22.8 implies the model is significant. There was only a $0.01 \%$ chance that a "model $F$ value" this large could occur due to noise. $\mathrm{p}$ values were found to be less than 0.0001 , less than 0.05 indicate model terms are significant. The "predicted R-squared" of 0.924 was in reasonable agreement with the "adjusted R-squared" of 0.967. Adeq Precision measures the signal to noise ratio, a ratio greater than four is desirable. The obtained ratio of 15.06 indicates an adequate signal, thus, the proposed model can be useful to navigate the design space.

Table 4: Summary of results of regression analysis for response parameters

\begin{tabular}{|c|c|c|c|c|c|}
\hline Models & $R^{2}$ & Adjusted $R^{2}$ & Predicted $R^{2}$ & Model F value & $p$ value \\
\hline \multicolumn{6}{|c|}{ Gelation temperature } \\
\hline Quadratic & 0.988 & 0.967 & 0.924 & 22.8 & 0.001 \\
\hline \multicolumn{6}{|c|}{ In vitro drug diffusion } \\
\hline Quadratic & 0.991 & 0.988 & 0.962 & 18.2 & 0.001 \\
\hline
\end{tabular}

Regression equations of fitted model

Gelation temperature $=+33-6.74 \mathrm{~A}-5.86 \mathrm{~B}+0 \mathrm{C}-0.85 \mathrm{AB}+0.075 \mathrm{AC}-0.025 \mathrm{BC}+1.47 \mathrm{~A}^{2}-1.78 \mathrm{~B}^{2}+0 \mathrm{C}^{2}+0 \mathrm{ABC}$

Drug diffusion $=+75.61-3.54 \mathrm{~A}-4.52 \mathrm{~B}+8.64 \mathrm{C}-3.1 \mathrm{AB}-0.175 \mathrm{AC}-1.3 \mathrm{BC}+7.32 \mathrm{~A}^{2}+5.94 \mathrm{~B}^{2}+0 \mathrm{C}^{2}+0 \mathrm{ABC}$

Where $\mathrm{A}=$ poloxamer $403, \mathrm{~B}=$ carbopol, $\mathrm{C}=$ fulvic acid 
For in vitro drug release response, the model $\mathrm{F}$ value of 18.2 indicated the model was significant. $p$ values were found to be 0.0001 , less than 0.05 , indicating model terms are significant. The "predicted R-squared" of 0.962 was in reasonable agreement with the "adjusted R-squared" of 0.988. Adeq Precision was obtained to be 11.44 indicating an adequate signal. Thus, the proposed model can be used to navigate the design space.

Since the values of $\mathrm{R}^{2}$ are quite high for both responses the polynomial equations form excellent fits to the experimental data and are highly statistically valid. Wide variation in gelation temperature for different runs ranging from 20 to $43.7^{\circ} \mathrm{C}$, indicates a strong influence of selected variables on it. This is also reflected by the wide range of values for coefficients of the terms of equation vi. The interaction terms show how the average gelation temperature and in vitro drug release changes when two variables are simultaneously changed. The negative coefficients for all three independent variables indicate an unfavorable effect on the response variables, while the positive coefficients for the interactions between two variables indicate a favorable effect while the

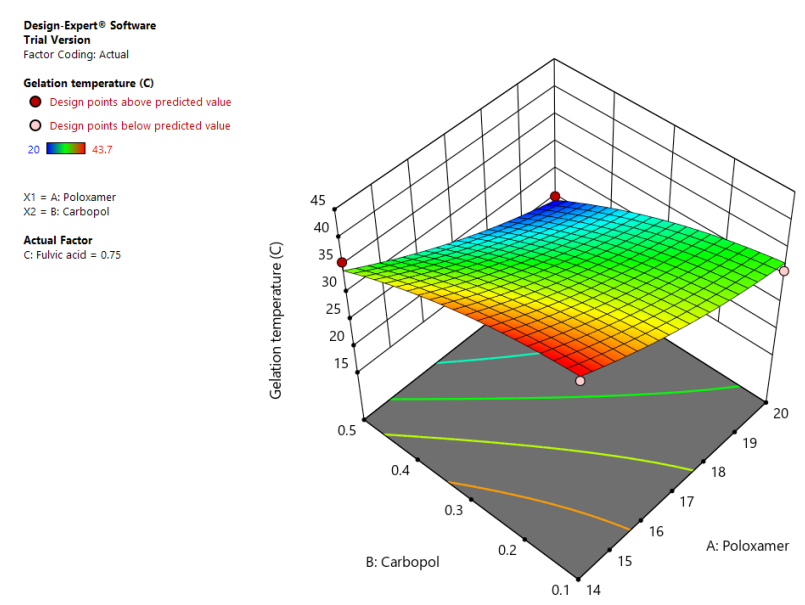

Fig. 3: 3D response surface plot showing influence of poloxamer and carbopol concentration on gelation temperature
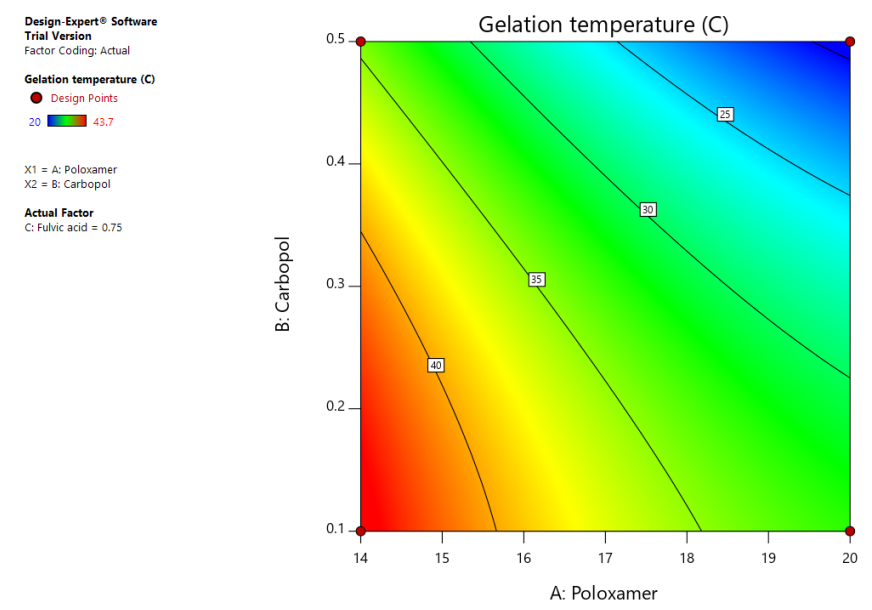

Fig. 4: Contour plot showing influence of poloxamer and carbopol concentration on gelation temperature small coefficients in equations VI and VII indicate that these terms contribute the least in the prediction of average gelation temperature and in vitro drug release. The value of the correlation coefficient $(r 2)$ of equation VII was found to be 0.988 , indicates a good correlation between independent and dependent variables. Among the independent variables selected and their interactions, only $\mathrm{X} 3$ was found to be significant $(\mathrm{p}<0.05)$, indicating a major contributing effect of $\mathrm{X} 3$ on in vitro drug release. A positive value of the coefficient for X3 (fulvic acid concentration) indicates a favorable effect on in vitro drug release.

\section{Response Surface Plots}

The relationship between the independent and dependent variables was further elucidated by constructing contour and three dimensional (3D) response surface plots. The effects of $\mathrm{X} 1, \mathrm{X} 2$, and $\mathrm{X} 3$ on response variables were shown in Figs 3 to 10.

\section{Search for Optimum Formulation}

When the outcomes of ANOVA were processed by the software using the criterion discussed earlier, an optimum
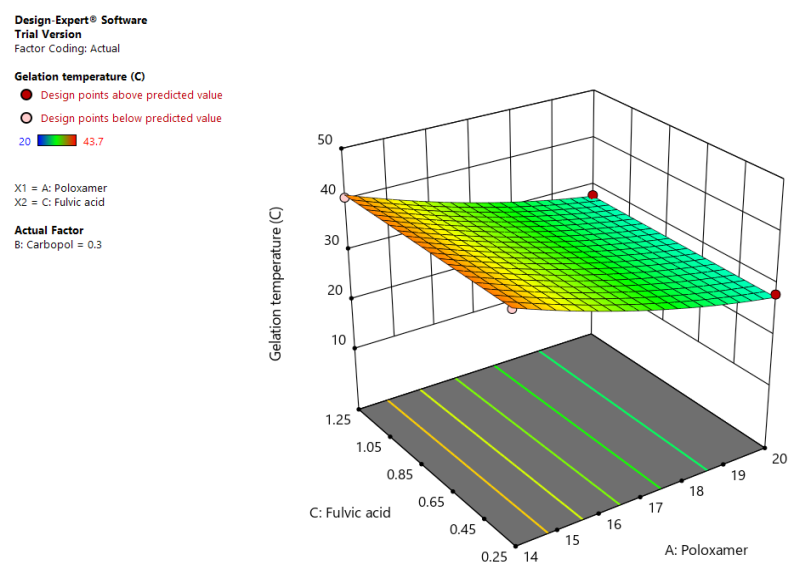

Fig. 5: 3D response surface plot showing influence of poloxamer and fulvic acid concentration on gelation temperature
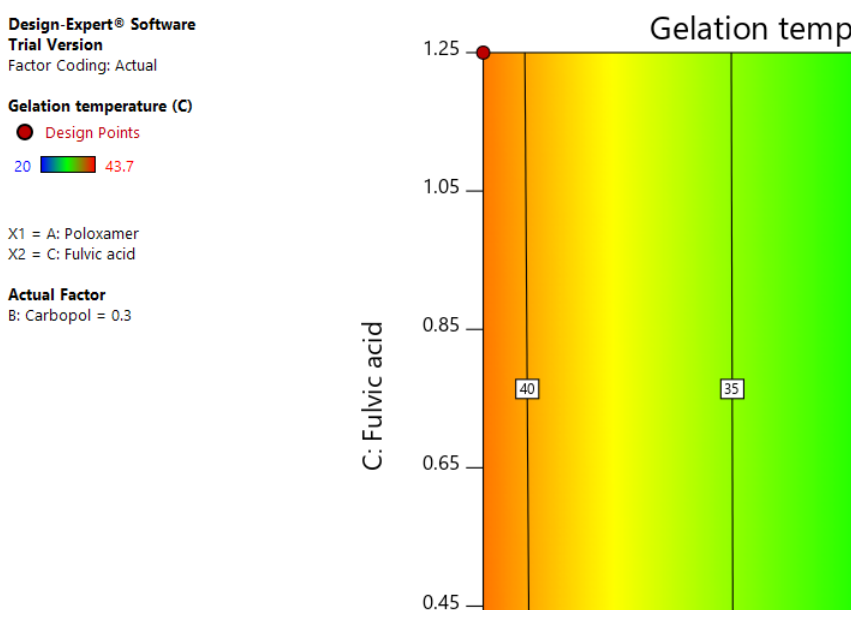

Fig. 6: Contour plot showing influence of poloxamer and fulvic acid concentration on gelation temperature 


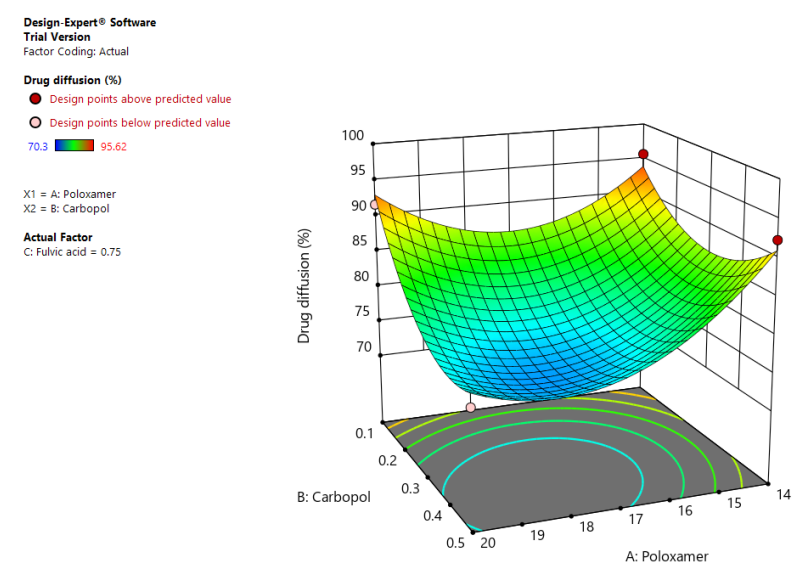

Fig. 7: 3D response surface plot showing influence of poloxamer and carbopol concentration on in vitro drug release

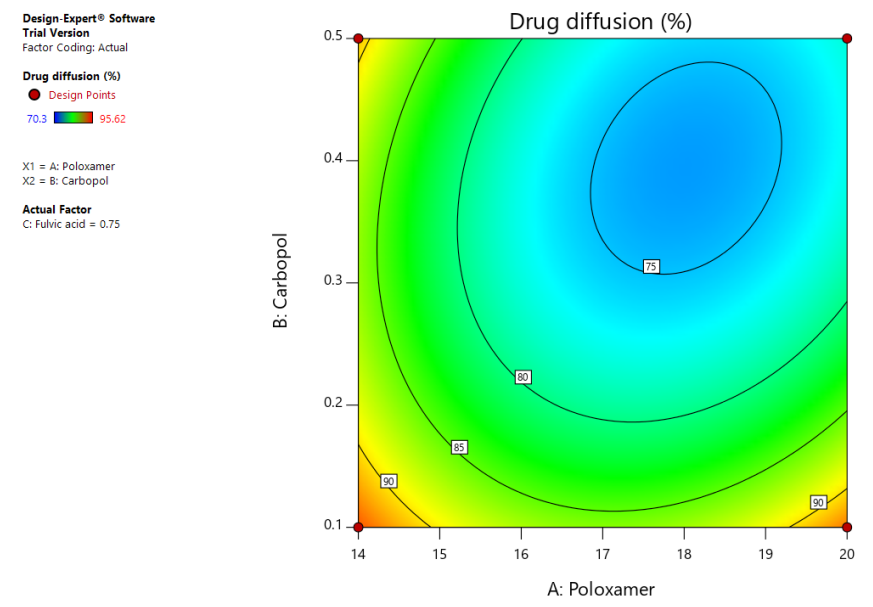

Fig. 8: Contour plot showing influence of poloxamer and carbopol concentration on in vitro drug release

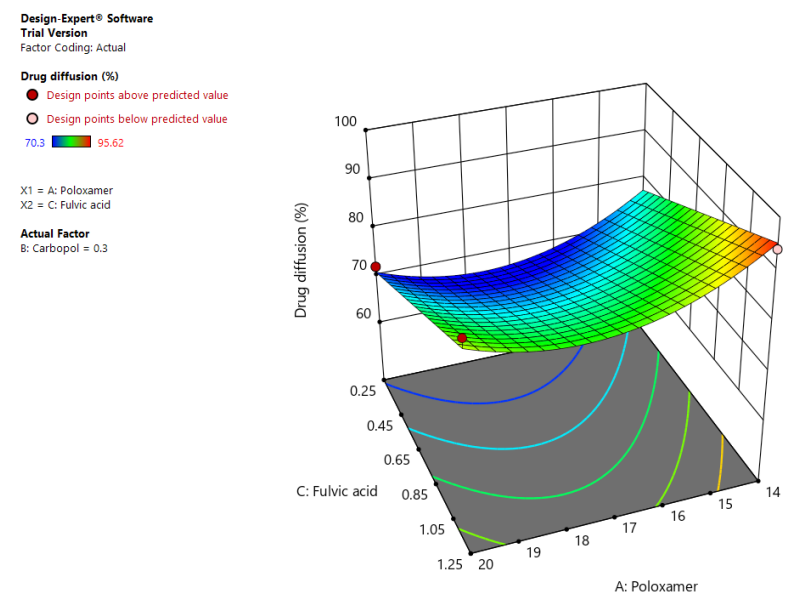

Fig. 9: 3D response surface plot showing influence of poloxamer and fulvic acid concentration on in vitro drug release formulation was obtained that contains $14.25 \% \mathrm{w} / \mathrm{v}$ of poloxamer, $0.49 \% \mathrm{w} / \mathrm{v}$ of carbopol, and $1.24 \% \mathrm{w} / \mathrm{v}$ of fulvic acid with a desirability of 0.99 . A value of the desirability function near to unity is generally preferred.

\section{Evaluation of Optimized In situ Nasal Gel of GRA}

The results of the evaluation of the optimized formulation are listed in Table 5. The predicted values of response parameters were found to be in close agreement with the observed ones.

\section{Histological Evaluation of Nasal Mucosa}

Fresh nasal mucosa treated with drug solution shows no destruction of epithelium membrane (Fig. 11), whereas nasal mucosa treated with phosphate buffer solution shows slight degradation of the epithelial membrane when observed under 100 and 400X (Fig. 12). Fresh nasal mucosa treated with optimized formulation shows slight degradation of the epithelial membrane, as well as, slight erosion and increase in vascularity at the basal membrane
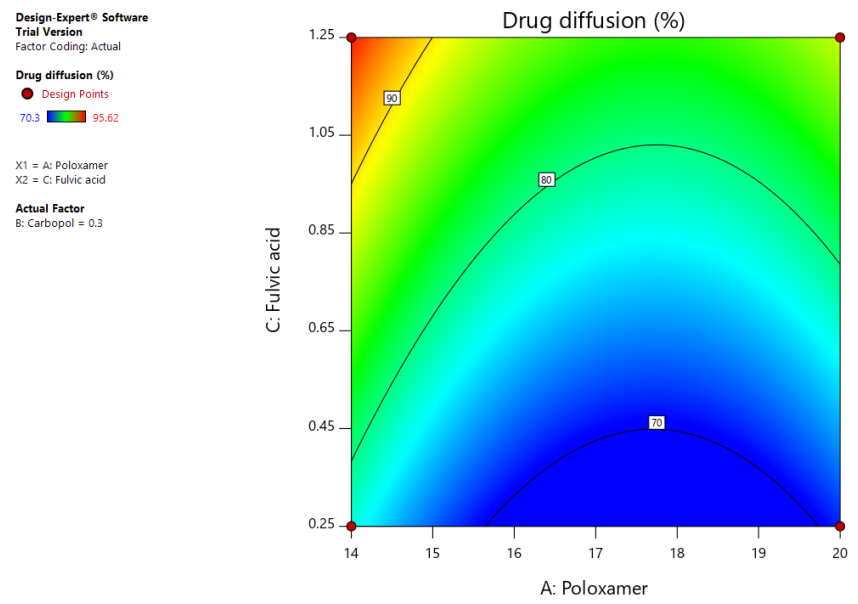

Fig. 10: Contour plot showing influence of poloxamer and fulvic acid concentration on in vitro drug release

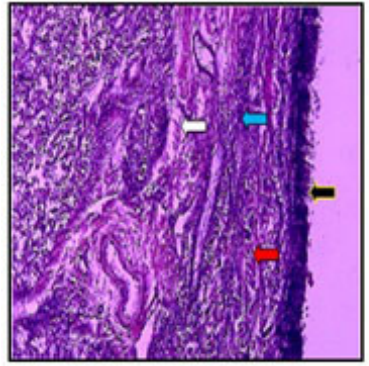

(A)

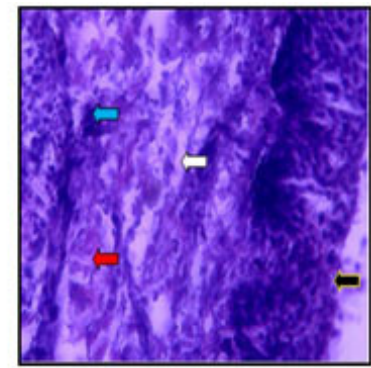

(B)
Fig. 11: Photograph of fresh nasal mucosa treated with drug solution showing cellular infiltration (blue arrow), destruction and necrosis of epithelium (black arrow), edema (white arrow), and vascular changes (red arrow); (A): 100X; (B): 400X

Table 5: Evaluation of optimized in situ nasal gel formulation

\begin{tabular}{lllllll}
\hline & & \multicolumn{2}{c}{ Gelation temp $\left({ }^{\circ} \mathrm{C}\right)$} & \multicolumn{3}{c}{ Ex vivo drug release $(\%)$} \\
\cline { 3 - 7 }$p H$ & Drug content $(\% w / v)$ & $(\mathrm{cm})$ & Predicted & Observed & Predicted & Observed \\
\hline 6.3 & 99.21 & 0.5 & 32.68 & 32.9 & 99.04 & 98.6 \\
\hline
\end{tabular}


Table 6: Data for plasma concentration profile of GRA after oral and nasal administration

\begin{tabular}{lll}
\hline Time $(\mathrm{min})$ & $\begin{array}{l}\text { Oral solution } \\
(\mathrm{ng} / \mathrm{mL})\end{array}$ & $\begin{array}{l}\text { In situ nasal gel } \\
(\mathrm{ng} / \mathrm{mL})\end{array}$ \\
\hline 0 & 0 & 0 \\
15 & 51.68 & 337.19 \\
30 & 86.57 & 410.12 \\
60 & 149.72 & 350.48 \\
90 & 190.19 & 234.25 \\
120 & 210.56 & 166.23 \\
150 & 152.36 & 120.39 \\
180 & 103.28 & 98.01 \\
210 & 65.28 & 90.56 \\
240 & 42.13 & 82.13 \\
\hline
\end{tabular}

Table 7: Mean pharmacokinetic parameter of GRA after oral and nasal administration

\begin{tabular}{lll}
\hline Parameters & Oral formulation & In situ nasal gel \\
\hline $\mathrm{C}_{\max }(\mathrm{ng} / \mathrm{mL})$ & 210.56 & 410.12 \\
$\mathrm{t}_{\max }(\mathrm{min})$ & 120 & 15 \\
$\mathrm{AUC}_{0 \rightarrow 4} \mathrm{ng} \cdot \mathrm{hr} / \mathrm{mL}$ & $29,496.68$ & $47,315.1$ \\
\hline
\end{tabular}

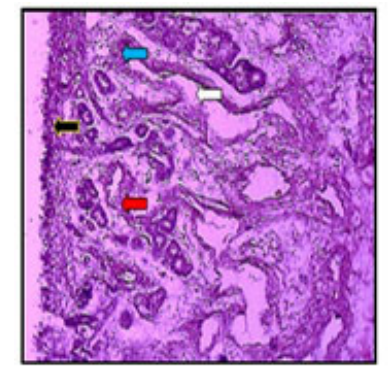

(A)

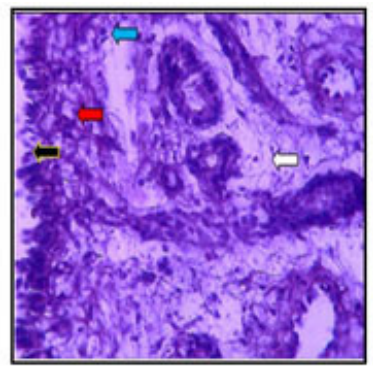

(B)
Fig. 12: Photograph of fresh nasal mucosa treated with phosphate buffer showing cellular infiltration (blue arrow), destruction and necrosis of epithelium (black arrow), edema (white arrow), and vascular changes (red arrow); (A): 100X; (B): 400X

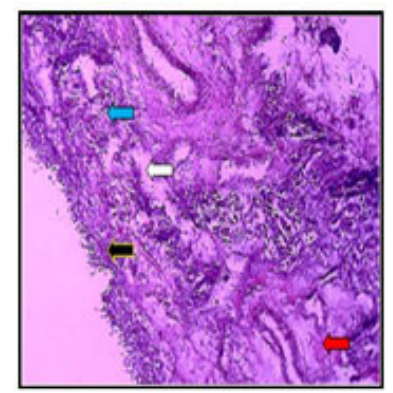

(A)

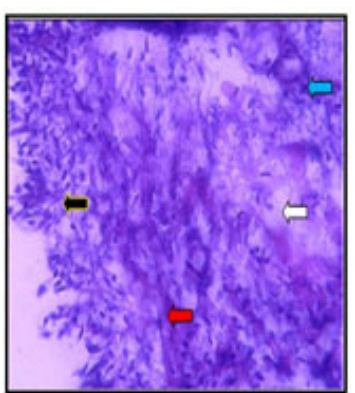

(B)
Fig. 13: Photograph of fresh nasal mucosa treated with optimized formulation showing cellular infiltration (blue arrow), destruction and necrosis of epithelium (black arrow), edema (white arrow), and vascular changes (red arrow); (A): 100X; (B): 400X

of the epithelial membrane when observed under 100 and 400X (Fig. 13). This effect was because of fulvic acid. Fulvic acid opens the tight junction of the epithelial membrane and increases the transport of the drug through the

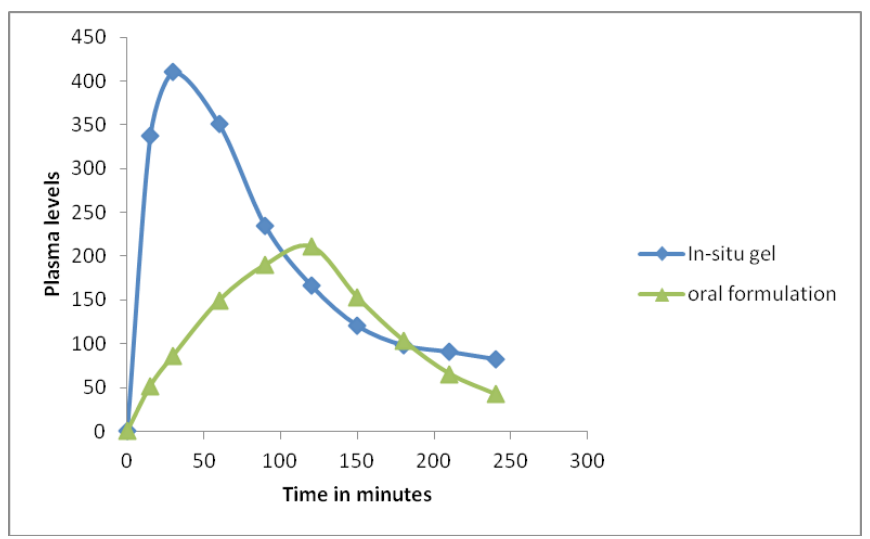

Fig. 14: Plasma concentration profile of granisetron after oral and nasal transmucosal administration

paracellular route. It also increases the vascularity at the basal membrane.

\section{In vivo Bioavailability Studies}

\section{Plasma Levels of GRA after Administration}

Plasma levels of GRA after administration of oral solution and in situ nasal gel are shown in Table 6. The mean pharmacokinetic parameters were calculated from the blood plasma concentration of the drug are shown in Table 7. Fig. 14 shows the blood plasma level profile of GRA after oral and nasal administration. It was observed that the plasma concentration profile of GRA in situ nasal gel showed enhancement in various quantitative parameters over oral formulation. Peak (maximum) plasma concentration $\left(\mathrm{C}_{\max }\right)$ of GRA in oral and in situ nasal gel was 210.56 and $410.12 \mathrm{ng} / \mathrm{mL}$, respectively. Whereas time $\left(\mathrm{t}_{\max }\right)$ to reach $\mathrm{C}_{\max }$ was 120 minutes for oral solution and 15 minutes for in situ nasal formulation. AUC0 $\rightarrow 4$ in oral solution and in situ nasal gel was 29,496.68 and 47,315.1 ng.hr/mL, respectively. These findings suggest that prepared in situ nasal gel showed a faster rate and enhanced extent of absorption of GRA as compared to the oral solution.

\section{Discussions}

In situ nasal gel formulations of GRA were prepared using the cold method as the triblock copolymers, like poloxamer are more soluble in water at cold temperatures. The $\mathrm{pH}$ of the formulations was maintained near nasal conditions. Lysozyme present in the nasal secretions is responsible for destroying certain microbes at acidic $\mathrm{pH}$. Under alkaline $\mathrm{pH}$, lysozyme is inactive and nasal tissue is susceptible to microbial infection. It is, therefore, advisable to keep the $\mathrm{pH}$ of the formulation in the range of 4.4 to 6.5. Ideally, the formulation should get converted from liquid to gel at nasal temperature, i.e., 32 to $34^{\circ} \mathrm{C}$. Hence, one of the criteria to search for the optimum formulation was matching this condition of gelation temperature. The spreadability of the formulations is desired to be lesser to prevent draining 
down the nasal cavity. With an increase in carbopol concentration, spreadability was observed to decrease. This observation was the result of the mucoadhesive properties of carbopol. The mucus membrane consists of an oligosaccharide side chain containing sialic acid and the carbopol contains $(-\mathrm{COOH}$ or $-\mathrm{OH})$ group. The $-\mathrm{COOH}$ or - $\mathrm{OH}$ group binds to the oligosaccharide side chain and mucoadhesion takes place.

As the concentration of fulvic acid increased the drug diffusion was increased drastically. Fulvic acid opens the tight junction of the epithelial membrane and increases the transport of the drug through paracellular routes. Fulvic acid also improves the vascularity at the basal membrane of the cell. In presence of fulvic acid, the permeation rate of GRA through nasal mucosa was increased, which is indicated by the significantly higher value of permeation coefficient and flux than in absence of penetration enhancer. So, it was concluded that fulvic acid has a property to enhance the permeability of GRA which is a Biopharmaceutical classification system (BCS) class III drug.

Design-Expert software was used to search the optimum combination of formulation variables to obtain maximum drug diffusion and gelation temperature near the nasal temperature. The models developed were found to be statistically significant on various parameters. A wide variation in the observed values of response variables indicated a strong influence of formulation variables on them. A good statistical agreement was also observed between these variables. The 3D response surface diagrams and their contour plots were further used to facilitate the understanding of the contribution of the variables and their interactions. It was observed that as the poloxamer concentration increases gelation temperature decreases and carbopol concentration had the same effect. But the magnitude of the effect was less as compared to poloxamer. There was no significant effect of fulvic acid concentration on gelation temperature. As the concentration of poloxamer and carbopol increases, the drug release decreases, while the magnitude of the effect was very large in the case of fulvic acid. The in vitro drug release was greatly increased with an increase in the concentration of fulvic acid. The true evaluation of the optimization models is to check its accuracy of predicting values of response variables practically. The optimized formulation showed very close agreement between predicted and observed values of drug discussion and gelation temperature. Hence, the model was found to be valid within the design space.

Plasma levels of GRA in rabbits after oral solution and in situ nasal were compared. A drastic increase in plasma levels of GRA after nasal administration over oral delivery suggests a promising approach for enhancing its bioavailability. Oral delivery of GRA faces issues, like less intestinal permeability, as well as, extensive hepatic first- pass metabolism. Nasal delivery bypasses this degradation by delivering the drug directly into the systemic circulation. The formulation discussed in this work is an in situ gel that gels at nasal temperature and has mucoadhesive properties. These features ensure an increase in retention time. Fulvic acid used as a penetration enhancer here helps in increasing transmucosal diffusion by opening tight junctions and enhancing paracellular transport.

\section{Conclusion}

It was observed that an optimum combination of poloxamer, carbopol, and fulvic acid can produce a formulation that gels at nasal temperature and enhances transmucosal permeation. This formulation is a nonviscous liquid at prescribed storage conditions and thus, offers ease of administration also. Plasma level profile and pharmacokinetic parameters of optimized in situ nasal gel were found to be enhanced quantitatively over oral formulation. Thus, it can be concluded that in situ nasal gel of GRA can enhance its bioavailability over oral delivery.

\section{REFERENCES}

1. Moffat AC, Osselton MD, Widdop . Clarke's Analysis of Drugs and Poisons. Edn 3, Pharmaceutical Press, London, 2004, pp. 1086 1087.

2. Dollery C. Therapeutic Drugs. Edn 2, Churchill Livingstone,1999, pp. G86-G90.

3. Sweetman SC. Martindale: The complete drug reference, Edn 35, Pharmaceutical Press, London, 2007, pp 1526-1602.

4. Prego C,García M, Torres D, Alonso MJ. Transmucosal macromolecular drug delivery. J Control Release. 2005;101(1-3):151-62.

5. Behl CR, Pimplaskar HK, Sileno AP, deMeireles J, Romeo VD. Effects of physicochemical properties and other factors on systemic nasal drug delivery. Adv Drug Deliv Rev.1998; 29, 89-116.

6. Cao Y, Zhang C, Shen W, Cheng Z, Yu LL, Ping Q. Poly (N-isopropylacrylamide) chitosan as thermosensitive in situ gelforming system for ocular drug delivery. J Control Release. 2007; Jul 31;120(3):186-194.

7. Wiilam AC, Barry BW. Penetration enhancers. Adv Drug Deliv Rev. 2004;56:603-618.

8. Mirza MA, Talegaonkar S, Ahmed FA, Iqbal Z. A novel and multifaceted excipient for vaginal drug delivery. J Excip Food Chem. 2001;2(4):98-112.

9. Kirsten Willis. An investigation of the effect of fulvic acid and humic acid on the absorption of selected drugs, vitamins and minerals using the iverted mouse gut model. M.S. Thesis, University of Pretoria. 2015.

10. Schmolka IR. Preparation and properties of Pluronic F127 gels for the treatment of burns. J Biomedical Mater Res. 1972;6:571-582.

11. Karnachi A, Khan M. Box-behnken design for the optimization of formulation variables of indomethacin coprecipitates with polymer mixtures. Int J Pharm. 1996;131(1):9-17.

12. Franz RM, Browne JE, Lewis A.R. Pharmaceutical dosage form: Disperse systems Vol. I, Marcel Dekker, NewYork.1998, pp. 427-519.

13. Angayer Kanchana S, Aruna A, Niraimath V, Jerad Suresh A. Spectrophotometric estimation of granisetron in bulk and tablet formulation. Int J Chem Sci. 2010;8(2):1169-1173.

14. Miler SC, Donovan MD. Effect of poloxamer 407 gels on the mioticactiviy of pilocarpine nitrates in rabbits. Int J Pharm. 1982;142-152.

15. Garala K, Joshi P, Shah M, Ramkishan A, Patel J. Formulation and evaluation of periodontal in situ gel. Int J Pharm Investig. 2013;3(1):29-41.

16. Pathan IB, Chudiwal V, Farooqui I, Shingare P. Formulation design 


\section{In situ Nasal Gel of Granisetron}

and evaluation of nasal in situ gel as a novel vehicle for Azelastine hydrochloride. Int J Drug Deliv. 2013;5:284-290.

17. Pinguet F, Bressolle F, Martel P, Salabert D, Astre C. Highperformance liquid chromatographic determination of granisetron in human plasma. Journal Chromatogr B. 1996;675:99-105.

18. Jiang Y, Lin M, Fan G, Chen Y, Zhen L, Zhao W, et al. Rapid determination of granisetron in human plasma by liquid chromatography coupled to tandem mass spectrometry and its application to bioequivalence study. J Pharm Biomed. 2006;42(4):464-473.
19. Barichello JM, Morishita M, Takayama K, Nagai T. Absorption of insulin from pluronic F-127 gels following subcutaneous administration in rats. Int J Pharm. 1999; Jul 20;184(2): 189-198.

20. Jeong B, Kim SW, Bae YH. Thermosensitive sol-gel reversible hydrogels. Adv Drug Deliv Rev. 2002;54(1):37-51.

21. Ricci EJ, Lunardi LO, Nanclares DM, Marchetti JM. Sustained release of lidocaine from Poloxamer 407 gels. Int J Pharm. 2005;288(2): 235-244.

HOW TO CITE THIS ARTICLE: Padalkar R, Madgulkar A. In situ nasal gel of granisetron for enhancement of bioavailability over oral delivery: formulation, optimization, and in vivo evaluation. Int. J. Pharm. Sci. Drug Res. 2020;12(5):544-553. DOI: 10.25004/IJPSDR.2020.120517 